\title{
MODULARITY BOUNDS FOR CLUSTERS LOCATED BY LEADING EIGENVECTORS OF THE NORMALIZED MODULARITY MATRIX
}

\author{
DARIO FASINO AND FRANCESCO TUDISCO
}

\begin{abstract}
Nodal theorems for generalized modularity matrices ensure that the cluster located by the positive entries of the leading eigenvector of various modularity matrices induces a connected subgraph. In this paper we obtain lower bounds for the modularity of that subgraph showing that, under certain conditions, the nodal domains induced by eigenvectors corresponding to highly positive eigenvalues of the normalized modularity matrix have indeed positive modularity, that is, they can be recognized as modules inside the network. Moreover we establish Cheeger-type inequalities for the cut-modularity of the graph, providing a theoretical support to the common understanding that highly positive eigenvalues of modularity matrices are related with the possibility of subdividing a network into communities.
\end{abstract}

Mathematics subject classification (2010): 05C50, 15A18, 15B99.

Keywords and phrases: Nodal domain, community detection, modularity, Cheeger inequality.

\section{REFERENCES}

[1] M. Bolla, Penalized versions of the Newman-Girvan modularity and their relation to normalized cuts and k-means clustering, Phys. Rev. E, 84 (2011), 016108.

[2] M. Bolla, B. Bullins, S. Chaturapruek, S. Chen, and K. Friedl, Spectral properties of modularity matrices, Linear Algebra Appl., 473 (2015), 359-376.

[3] F. Chung, Spectral Graph Theory, CBMS Regional Conference Series in Mathematics, 92, AMS, Providence, 1997.

[4] F. ChUng AND R. GRAham, Quasi-random graphs with given degree sequences, Random Structures Algorithms, 32 (2008), 1-19.

[5] E. B. Davies, G. M. L. Gladwell, J. Leydold, And P. F. Stadler, Discrete nodal domain theorems, Linear Algebra Appl., 336 (2001), 51-60.

[6] F. De Montgolfier, M. Soto, And L. Viennot, Asymptotic modularity of some graph classes, In: Algorithms and computation, vol. 7074 of Lecture Notes in Comput. Sci., pages 435-444. Springer, Heidelberg, 2011.

[7] A. M. Duval And V. Reiner, Perron-Frobenius type results and discrete versions of nodal domain theorems, Linear Algebra Appl., 294 (1999), 259-268.

[8] D. FASINO AND F. TUdisco, An algebraic analysis of the graph modularity, SIAM J. Matrix Anal. Appl., 35 (2014), 997-1018.

[9] D. FASINO AND F. Tudisco, Generalized modularity matrices, Linear Algebra Appl., 502 (2016), 327-345.

[10] M. FIEDLER, Algebraic connectivity of graphs, Czechoslovak Mathematical Journal, 23 (1973), 298305.

[11] M. FIEDLER, A property of eigenvectors of nonnegative symmetric matrices and its application to graph theory, Czechoslovak Mathematical Journal, 25 (1975), 619-633.

[12] S. Fortunato, Community detection in graphs, Physics Reports, 486 (2010), 75-174.

[13] A. Kehagias And L. Pitsoulis, Bad communities with high modularity, Eur. Phys. J. B, 86 (2013), Art. 330.

[14] S. MajStorovic And D. STEVAnOvic, A note on graphs whose largest eigenvalues of the modularity matrix equals zero, Electron. J. Linear Algebra, 27 (2014), 611-618. 
[15] M. E. J. Newman, Finding community structure in networks using the eigenvectors of matrices, Phys. Rev. E, 74 (2006), 036104.

[16] M. E. J. Newman, Networks: An Introduction, OUP Oxford, Oxford, 2010.

[17] M. E. J. Newman AND M. GiRvan, Finding and evaluating community structure in networks, Phys. Rev. E, 69 (2004), 026113.

[18] D. L. Powers, Graph partitioning by eigenvectors, Linear Algebra Appl., 101 (1988), 121-133.

[19] S. E. SCHAEFFER, Graph clustering, Computer Science Review, 1 (2007), 27-64.

[20] J. H. Wilkinson, The Algebraic Eigenvalue Problem, Oxford University Press, Oxford, 1965. 